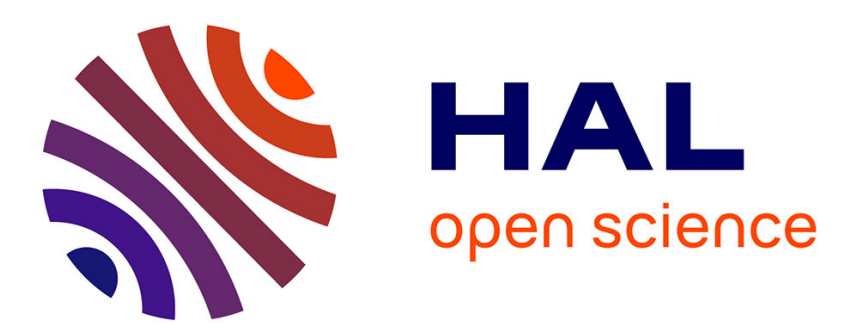

\title{
Femur Specific Polyaffine Model to Regularize the Log-Domain Demons Registration
}

Christof Seiler, Xavier Pennec, Lucas Ritacco, Mauricio Antonio Reyes

Aguirre

\section{- To cite this version:}

Christof Seiler, Xavier Pennec, Lucas Ritacco, Mauricio Antonio Reyes Aguirre. Femur Specific Polyaffine Model to Regularize the Log-Domain Demons Registration. Proceedings of SPIE Medical Imaging '11, 2011, Lake Buena Vista, Floride, United States. Paper 7962-15, 10.1117/12.878316 . inria-00616216

\section{HAL Id: inria-00616216 \\ https://hal.inria.fr/inria-00616216}

Submitted on 28 Jun 2013

HAL is a multi-disciplinary open access archive for the deposit and dissemination of scientific research documents, whether they are published or not. The documents may come from teaching and research institutions in France or abroad, or from public or private research centers.
L'archive ouverte pluridisciplinaire HAL, est destinée au dépôt et à la diffusion de documents scientifiques de niveau recherche, publiés ou non, émanant des établissements d'enseignement et de recherche français ou étrangers, des laboratoires publics ou privés. 


\title{
Femur Specific Polyaffine Model to Regularize the Log-Domain Demons Registration
}

\author{
Christof Seiler $^{a, b}$, Xavier Pennec ${ }^{b}$ Lucas Ritacco $^{c, d}$ and Mauricio Reyes ${ }^{a}$ \\ ${ }^{a}$ Institute for Surgical Technology and Biomechanics, University of Bern, Switzerland; \\ ${ }^{b}$ Asclepios Project, INRIA Sophia Antipolis, France; \\ ${ }^{c}$ Department of Medical Informatics and Institute of Orthopedics Carlos E. Ottolenghi, \\ Buenos Aires, Argentina; \\ ${ }^{d}$ Italian Hospital of Buenos Aires, Argentina
}

\begin{abstract}
Osteoarticular allograft transplantation is a popular treatment method in wide surgical resections with large defects. For this reason hospitals are building bone data banks. Performing the optimal allograft selection on bone banks is crucial to the surgical outcome and patient recovery. However, current approaches are very time consuming hindering an efficient selection. We present an automatic method based on registration of femur bones to overcome this limitation. We introduce a new regularization term for the log-domain demons algorithm. This term replaces the standard Gaussian smoothing with a femur specific polyaffine model. The polyaffine femur model is constructed with two affine (femoral head and condyles) and one rigid (shaft) transformation. Our main contribution in this paper is to show that the demons algorithm can be improved in specific cases with an appropriate model. We are not trying to find the most optimal polyaffine model of the femur, but the simplest model with a minimal number of parameters. There is no need to optimize for different number of regions, boundaries and choice of weights, since this fine tuning will be done automatically by a final demons relaxation step with Gaussian smoothing. The newly developed synthesis approach provides a clear anatomically motivated modeling contribution through the specific three component transformation model, and clearly shows a performance improvement (in terms of anatomical meaningful correspondences) on $146 \mathrm{CT}$ images of femurs compared to a standard multiresolution demons. In addition, this simple model improves the robustness of the demons while preserving its accuracy. The ground truth are manual measurements performed by medical experts.
\end{abstract}

Keywords: Registration, Regularization, Log-Domain Demons, Polyaffine, Femur, Allotransplantation

\section{INTRODUCTION}

Tumor excision is the primary treatment of aggressive or recurrent benign bone tumors and malignant bone sarcomas. This requires an invasive surgical intervention that entails a residual bone defect, which can be reconstructed with a fresh frozen bone allotransplantation. In orthopedics, recent improvements in diagnostic and therapeutic techniques have produced an increase of the patient survival as well as a reduction of complication rate. ${ }^{1}$ It has been shown that the selection of bone allograft in terms of shape and size is crucial to prevent changes in joint mobility and load distribution, which can lead to joint fractures and early joint degeneration. ${ }^{2}$ However, current selection approaches are very time-consuming, mostly based on manual measurements performed directly on the bones or on three-dimensional models reconstructed from images.

Ideally, an automatic allograft selection system could perform shape comparisons between the patient's anatomy and the complete bone bank. In this case, it would be necessary to allocate extra time to the clinical work flow in order to segment the patient's anatomy and register it to the existing bone bank.

Practically, to avoid preprocessing, hence reducing preoperative planning time, the authors in Ritacco et al. ${ }^{3}$ proposed a simplified protocol for bone size characterization. The so-called ABC protocol is based on well-known anatomical landmarks which can be extracted from the CT image and used in a selection criteria. The protocol defines 6 landmarks

Further author information: (Send correspondence to C. Seiler)

C. Seiler: E-mail: christof.seiler@istb.unibe.ch 
on the distal femur: Transepicondyle distance (A), anterior-posterior distance in the medial condyle (B) and in the lateral condyle (C). One possible automation of this protocol requires two steps. First, CT images of all donor bones are segmented and registered to a preselected reference. The ABC measurements can now be performed on the reference and correspondences in the other images can be extracted and stored. Second, the surgeon identifies manually the ABC landmarks on the patient CT image and uses the previously stored ABC measures to select the best match.

In order to automatize the procedure, we first tried to register non-linearly the CT image to the candidate bones using a demons registration approach. ${ }^{4}$ However, we observed that the direct use of this method failed to capture torsion and rotation in head and condyle regions of the femur bone. We propose in this paper to model the expected deformations in the specific case of femur bone registration considering torsion and rotation in head and condyles, and to use this femur specific polyaffine model as an adapted regularization prior for the demons registration in replacement of the uninformed Gaussian smoothing.

We build on the concept of polyaffne registration, introduced in Arsigny et al. ${ }^{5}$ and fully developed into an efficient and practical framework in Commowick et al. ${ }^{6}$ We initialize the demons with a polyaffine registration of three regions (head, shaft and condyles) using block matching ${ }^{7}$ and regularize the subsequent iterations with a polyaffine model integrated into the symmetric demons algorithm. As a last step, we relax the polyaffine constraint to allow more general local deformations regularized using a Gaussian smoothing.

The pipeline was developed using open source libraries: VTK, ITK and Qt (UI framework). In the following, we develop the methodology and show comparison results between multiresolution demons with Gaussian regularization and our approach considering demons with the new polyaffine regularization term.

\section{A POLYAFFINE LOG-DOMAIN DEMONS FOR THE FEMUR}

Our main contribution in this paper is to show that the demons algorithm can be improved in specific cases with an appropriate model. The main idea of the proposed algorithm is to introduce a polyaffine regularization of the velocity field. The polyaffine model divides the femur in three regions to capture torsion and rotation in head and condyles. In the following sections we review the two methods that we later combine: Log-domain and polyaffine registration. We show how to add our specific femur model during the regularization step of demons through an estimate of the polyaffine parameters given a velocity field. This estimate replaces the standard Gaussian smoothing.

\subsection{Log-Domain Registration}

To setup correspondences between anatomical images, a set of images are registered to a reference. We use the novel symmetric diffeomorphic demons registration approach described in Vercauteren et al. ${ }^{4}$ What is new in this registration framework is the efficient optimization in the log-domain. As a consequence, the results of the registration are stationary velocity fields. These velocity fields can be looked at as generators for diffeomorphic deformations through the group exponential map that can be very efficiently computed using the scaling and squaring method. ${ }^{8}$

\subsection{Polyaffine Registration}

In Commowick et al. ${ }^{6}$ the authors introduced an iterative framework to fuse regional affine transformation to a global transformation. In a first step, an affine registration is performed for each region, second, the affine transformation are regularized, and finally a global transformation is calculated through a weighted average of all regions. The averaging of affine transformations can be done on the matrix logarithms to ensure invertibility of the final transformation. The regions are manually defined on the reference prior to the registration procedure.

\subsection{Femur Polyaffine Model}

For the polyaffine femur model we define three regions on the reference image. The middle section of the femur is defined as shaft region. The shaft region is centered in the middle of the reference femur and covers half of the volume of the image. The distal and the proximal end are condyle and head region, respectively. All images to be registered are anisotropically scaled to fit the size of the reference femur before starting with the polyaffine registration.

Let $M_{i}$ be the $3 \times 4$ non null components of the log of the affine transformation

$$
\log \left(\left[\begin{array}{cc}
A_{i} & t_{i} \\
0 & 1
\end{array}\right]\right)=\left[\begin{array}{c}
M_{i} \\
0
\end{array}\right],
$$


where $i=1$ is an affine transform for condyles, $i=2$ a rigid transform for the shaft and $i=3$ an affine transform for the head region. We use the following weight function to connect the transformations:

$$
w_{i}(x)=f\left(\frac{e_{z}^{T} \cdot\left(x-x_{i}\right)}{\left\|x_{3}-x_{1}\right\|}\right)
$$

where $f(\cdot)$ is a Gaussian function of the spatial position $x \in \mathbb{R}^{3}$ with $\mu=0$ and $\sigma=0.125$. The Gaussian parameters are defined heuristically with the aim to find the best compromise between smooth transitions from one region to the next and considering each region individually with the least amount of influence by others. The center positions of each region $x_{1}, x_{2}$ and $x_{3}$ are set to the center of the transverse plane. Along the transversal axis the positions are set to achieve an equal weighting at the boundary of two adjacent regions. The weights are normalized to $\sum w_{i}(x)=1$. Thus the velocity field predicted by our model is

$$
v_{\text {poly }}(x)=w_{1}(x) \cdot M_{1} \cdot x+w_{2}(x) \cdot M_{2} \cdot x+w_{3}(x) \cdot M_{3} \cdot x
$$

\subsection{Estimation of Femur Polyaffine Model}

Here we introduces the estimation of our polyaffine model which will be needed in the next section for the demons regularization. We show how to find a closed form solution on logarithms of affine transformations. Given a velocity field, the log affine parameters of (3) can be estimated by solving a linear least square problem. The least square error with respect to the velocity field observed is given by:

$$
C\left(M_{1}, M_{2}, M_{3}\right)=\int d(x)\left\|w_{1}(x) \cdot M_{1} \cdot x+w_{2}(x) \cdot M_{2} \cdot x+w_{3}(x) \cdot M_{3} \cdot x-v(x)\right\|^{2} d x
$$

where $d(x)$ is a binary variable set to 0 in the background. Using the Frobenius inner product which generalizes the dot product to matrices, $\|W\|^{2}=\operatorname{Tr}\left(W . W^{T}\right)$, we obtain the following directional derivative:

$$
\begin{aligned}
\partial_{W} C & =\lim _{\epsilon \rightarrow 0} \frac{C\left(M_{1}+\epsilon W_{1}, M_{2}+\epsilon W_{2}, M_{3}+\epsilon W_{3}\right)-C\left(M_{1}, M_{2}, M_{3}\right)}{\epsilon} \\
& =\int d(x) \cdot \operatorname{Tr}\left[\left(w_{1}(x) \cdot W_{1} \cdot x+w_{2}(x) \cdot W_{2} \cdot x+w_{3}(x) \cdot W_{3} \cdot x\right)\left(w_{1}(x) \cdot M_{1} \cdot x+w_{2}(x) \cdot M_{2} \cdot x+w_{3}(x) \cdot M_{3} \cdot x-v(x)\right)^{T}\right] \\
& =\operatorname{Tr}\left[W_{1} \cdot \int d(x) \cdot w_{1}(x) \cdot x \cdot\left(w_{1}(x) \cdot M_{1} \cdot x+w_{2}(x) \cdot M_{2} \cdot x+w_{3}(x) \cdot M_{3} \cdot x-v(x)\right)^{T}\right]+\text { same for } W_{2} \text { and } W_{3} \cdot
\end{aligned}
$$

At the optimum, the directional derivative should be null in each direction $W$, i.e. $\partial_{W} C=0$ for all matrix $\mathrm{W}$. As we have $\operatorname{Tr}(W . A)=0$ for all $W$ iff $A=0$, we end up with the system

$$
\sum_{j} M_{i} \cdot \Sigma_{i j}=B_{i}
$$

with

$$
\begin{aligned}
\Sigma_{i j} & =\int d(x) \cdot w_{i}(x) \cdot w_{j}(x) \cdot x \cdot x^{T} d x, \\
B_{i} & =\int d(x) \cdot w_{i}(x) \cdot v(x) \cdot x^{T} d x .
\end{aligned}
$$

To estimate $M_{1}, M_{2}$ and $M_{3}$ we need to solve the following system:

$$
\left[\begin{array}{lll}
M_{1} & M_{2} & M_{3}
\end{array}\right]\left[\begin{array}{lll}
\Sigma_{11} & \Sigma_{12} & \Sigma_{13} \\
\Sigma_{12} & \Sigma_{22} & \Sigma_{23} \\
\Sigma_{13} & \Sigma_{23} & \Sigma_{33}
\end{array}\right]=\left[\begin{array}{lll}
B_{1} & B_{2} & B_{3}
\end{array}\right]
$$

where $\Sigma$ is symmetrical and thus diagonalizable and the solution is given by:

$$
M=B \cdot \Sigma^{-1} .
$$

This system can be solved very efficiently since the matrix $\Sigma$ is only of dimension $12 \times 12$. $\Sigma$ does not change for different velocity field observations as long as the weighting functions are fixed, hence only $B$ needs to be recomputed. This fact will be helpful during the iterative regularization of demons which is described in the next section. 
Algorithm 1 Relaxed Femur Polyaffine Log-domain Demons (RFPLD)

- Affine registration of condyles, shaft and head region

- Combine affine transformations to one velocity field $v$ using (3)

- Initialize demons with $v$

- Iterate until convergence:

- Compute the demons forces $u$

- Let $v \leftarrow Z_{X}(v, u)$ (Baker-Campbell-Hausdorff approximation ${ }^{4}$ )

- For polyaffine regularization let $v \leftarrow$ solve (6) for $v$ and combine $M_{1}, M_{2}$ and $M_{3}$ using (3)

- Iterate until convergence:

- Compute the demons forces $u$

- Let $v \leftarrow Z_{X}(v, u)$

- For diffusion-like regularization let $v \leftarrow K_{\operatorname{diff}} \star v$ (Gaussian smoothing)

\subsection{Regularization with a Femur Polyaffine Model}

Since the demons algorithm decouples the registration problem in force computations using optical flow equations and a smoothing step it is straight forward to introduce a new regularization by replacing the latter. Our new polyaffine regularized demons estimates the polyaffine femur model at each step of the demons registration, see Algorithm 1, and thus connects the non-linear and image intensity based computation of forces with the femur model.

\section{VALIDATION WITH ABC GROUND TRUTH AND VISUALIZATION OF TORSION AND ROTATION}

We compare three different registration methods: First, a standard multiresolution demons with Gaussian smoothing for the regularization, referenced hereafter with Standard Log-domain Demons (SLD). Second, the newly developed polyaffine regularized demons, referenced hereafter with Femur Polyaffine Log-domain Demons (FPLD). Third, the second registration method with a subsequent relaxation of the regularization term to Gaussian smoothing as described in Algorithm 1, referenced hereafter with Relaxed Femur Polyaffine Log-domain Demons (RFPLD). We conduct a task specific validation of the newly developed regularization, testing the performance of all three registration methods on $\mathrm{ABC}$ measurements for bone tumor replacement. The ground truth of the measurements was obtained by manually selecting the landmarks on 3D reconstructed surface models. Two medical doctors performed these measurements independently and one expert repeated the measurements after a few days to yield the interobserver variability.

The results in Fig. 1 show that for the SSD only a small difference is visible for SLD and RFPLD, whereas FPLD is clearly higher. The smoothness decreases slightly in our new approach. The newly developed method RFPLD provides smaller ABC measurement errors compared to the standard demons. In two of three cases the FPLD shows smaller ABC measurement errors than the old approach. The accuracy of the A and B measurements (and in a smaller measure for C) are of the same order of magnitude than the intra and inter-rater errors for our relaxed polyaffine model.

To analyze the obtained results we perform a principle component analysis (PCA) on the velocity fields obtained by the three registration methods. In Fig. 2 the variance along the first three PCs of the six landmarks are visualized with curves. Each curve corresponds to the flow of a landmark point by integrating the velocity field starting at the mean $(t=0)$ in both direction with end points $t=3 \sigma$ and $t=-3 \sigma$. These curves are also called streamlines and used in fluid dynamics. Since the exponential map and streamlines of a flow are equivalent, it is an intrinsic visualization tool to understand the spatial deformations.

Similarly, to show the torsion and rotation variance in the femur, streamlines were calculated for the femoral head (Fig. 3). Two different views were chosen to best visualize the variability patterns. It can clearly be seen that the first $\mathrm{PC}$ describes rotations along the transversal axis, the second $\mathrm{PC}$ a change of the caput collum diaphysis angle and the third PC a S-shaped deformation pattern with a stretching of the femoral head. 

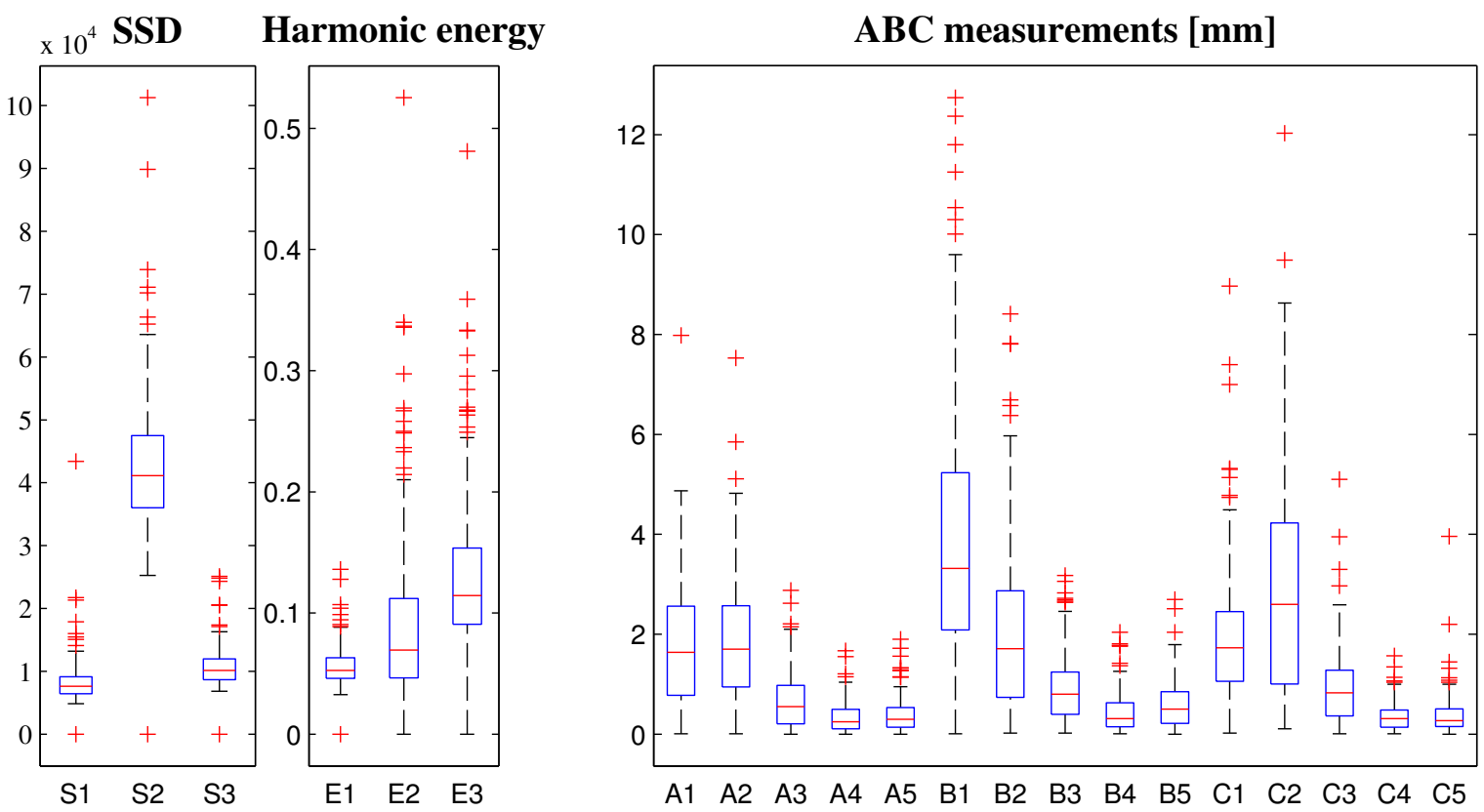

Figure 1. Boxplots comparing the three registration methods: SLD $(\cdot 1)$, FPLD $(\cdot 2)$ and RFPLD $(\cdot 3)$. (.4) and $(\cdot 5)$ are interobserver and intraobserver errors. (S.), (E.), (A.), (B.) and (C.) are SSD, harmonic energy and the three ABC distances.

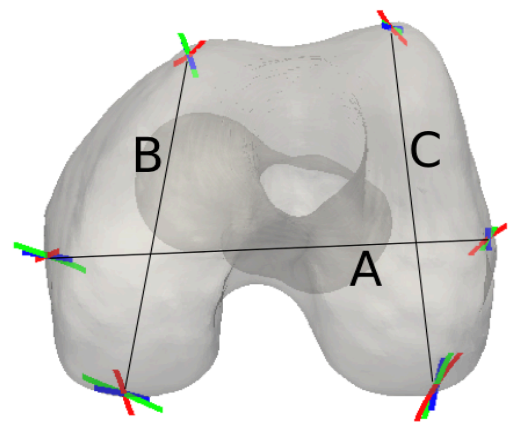

(a)

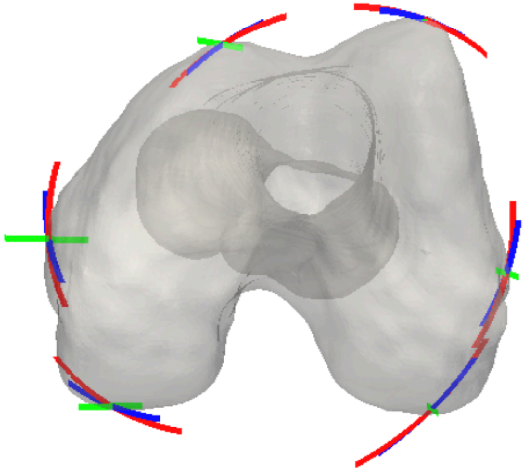

(b)

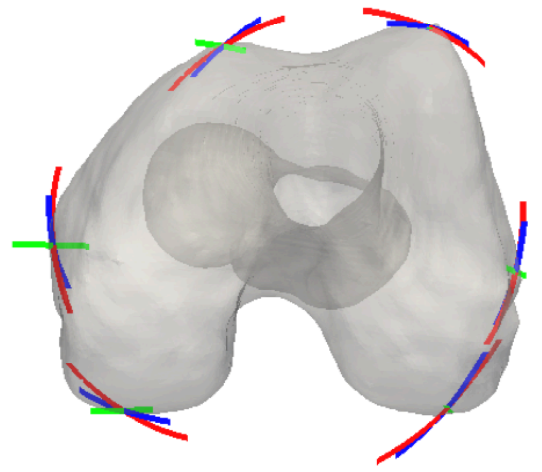

(c)

Figure 2. Visualization of the variance of the velocity fields obtained by the three registration methods, (a) SLD (b) FPLD. (c) RPLD, shown here are only the six ABC landmarks points, which are integrated from $t=-3 \sigma$ to $t=3 \sigma$. The first three PCs: Red, green and blue are PC1, PC2 and PC3, respectively. On (a) ABC distances are illustrated: Transepicondyle distance (A), anterior-posterior distance in the medial condyle (B) and in the lateral condyle (C).

\section{DISCUSSION AND CONCLUSIONS}

In this work we introduced a femur specific polyaffine model to replace the regularization step of the demons algorithm. The goal was to improve the registration in terms of anatomical correspondences to enable automatic $\mathrm{ABC}$ measurements for allograft selection.

Comparisons to the standard demons clearly indicate the power of the new method to capture the anatomical variability. This was visualized in Fig. 2 with the much higher variances at landmark positions. The validation in Fig. 1 shows that our method provides higher or equal SSD but better ABC correspondences suggesting that the standard demons finds a local minimum. Instead our method guides the demons to a minimum that is anatomically more meaningful in terms of correspondences. The ABC measurements give us a ground truth that is clinically relevant. The accuracy of the measurements are of the same order of magnitude than the intra and inter-rater errors for our relaxed polyaffne model, 


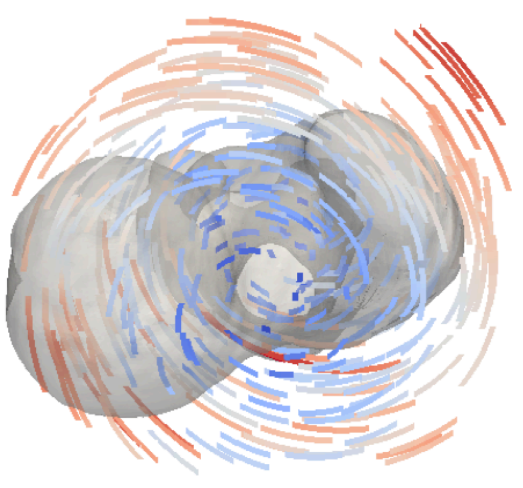

(a)

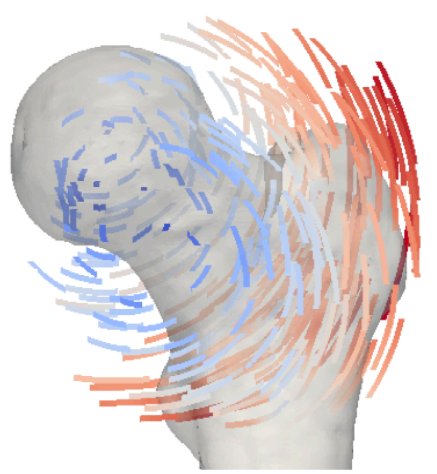

(b)

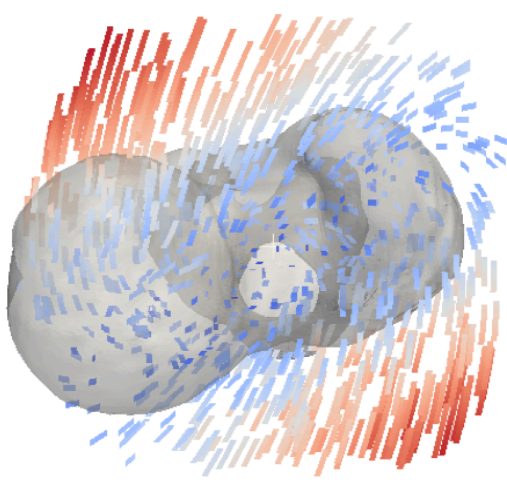

(c)

Figure 3. Visualization of the variance of the velocity fields through streamlines. The first three PCs of RFPLD is shown for randomly picked points which are integrated from $t=-3 \sigma$ to $t=3 \sigma$ : (a) PC1, (b) PC2 and (c) PC3. The color describes the length of the trajectory.

which shows that we are clearly reaching the performances of the human raters. This indicates that it is important to include our intuitive understanding of the anatomy and shows that in specific cases the demons can be improved by incorporating a simple a priori model like our femur specific polyaffine model.

Future work includes changing the resampling of the images from $1.25 \mathrm{~mm}$ to a smaller voxel spacing, to further improve the accuracy thus potentially being more accurate than the human raters. The demons provides a straight forward and fast vehicle for the connection of models with medical images since it divides force computations and the regularization step. This opens up new ways of model-based registration with the demons algorithm for other anatomical structures.

\section{REFERENCES}

[1] Muscolo, D. L., Ayerza, M. A., Aponte-Tinao, L. A., and Ranalletta, M., "Use of Distal Femoral Osteoarticular Allografts in Limb Salvage Surgery," J Bone Joint Surg Am 87, 2449-2455 (November 2005).

[2] Enneking, W. F. and Campanacci, D. A., "Retrieved Human Allografts: A Clinicopathological Study," J Bone Joint Surg Am 83, 971-986 (July 2001).

[3] Ritacco, L. E., Orias, A. E., Tinao, L. A., Muscolo, D. L., Bernaldo de Quirós, F. G., and Nozomu, I., "ThreeDimensional Morphometric Analysis of the Distal Femur: A Validity Method for Allograft Selection Using a Virtual Bone Bank," in [Medinfo], (September 2010).

[4] Vercauteren, T., Pennec, X., Perchant, A., and Ayache, N., "Symmetric Log-Domain Diffeomorphic Registration: A Demons-Based Approach," in [Medical Image Computing and Computer-Assisted Intervention MICCAI 2008], Metaxas, D., Axel, L., Fichtinger, G., and Székely, G., eds., Lecture Notes in Computer Science 5241, 754-761, Springer (2008).

[5] Arsigny, V., Pennec, X., and Ayache, N., "Polyrigid and Polyaffine Transformations: A Novel Geometrical Tool to Deal with Non-rigid Deformations Application to the Registration of Histological Slices," Medical Image Analysis 9, 507-523 (December 2005).

[6] Commowick, O., Arsigny, V., Isambert, A., Costa, J., Dhermain, F., Bidault, F., Bondiau, P.-Y., Ayache, N., and Malandain, G., "An Efficient Locally Affine Framework for the Smooth Registration of Anatomical Structures," Medical Image Analysis 12, 427-441 (August 2008).

[7] Ourselin, S., Roche, A., Prima, S., and Ayache, N., "Block Matching: A General Framework to Improve Robustness of Rigid Registration of Medical Images," in [Medical Image Computing and Computer-Assisted Intervention (MICCAI)], Delp, S., DiGoia, A., and Jaramaz, B., eds., Lecture Notes in Computer Science 1935, 557-566, Springer (2000).

[8] Arsigny, V., Commowick, O., Pennec, X., and Ayache, N., "A Log-Euclidean Framework for Statistics on Diffeomorphisms," in [Medical Image Computing and Computer-Assisted Intervention (MICCAI)], Larsen, R., Nielsen, M., and Sporring, J., eds., Lecture Notes in Computer Science 4190, 924-931, Springer (2006). 